\title{
Exogenous glucagon-like peptide-2 improves outcomes of intestinal adaptation in a distal-intestinal resection neonatal piglet model of short bowel syndrome
}

\author{
Megha Suri' ${ }^{1}$, Justine M. Turner ${ }^{2}$, David L. Sigalet ${ }^{3}$, Pamela R. Wizzard ${ }^{2}$, Patrick N. Nation ${ }^{4}$, Ron O. Ball ${ }^{5}$, Paul B. Pencharz ${ }^{6-8}$, \\ Patricia L. Brubaker ${ }^{9,10}$ and Paul W. Wales ${ }^{1,2,6}$
}

BACKGROUND: Endogenous glucagon-like peptide-2 (GLP-2) levels and intestinal adaptation are reduced in distal-intestinal resection animal models of short bowel syndrome (SBS) that lack remnant ileum. We hypothesized that exogenous GLP-2 would improve intestinal adaptation in a distal-intestinal resection neonatal piglet model of SBS.

METHODS: In all, 35 piglets were randomized to 2 treatment and 3 surgical groups: control (sham), $75 \%$ mid-intestinal resection (JI), and 75\% distal-intestinal resection (JC). Parenteral nutrition (PN) commenced on day 1 and was weaned as enteral nutrition (EN) advanced. IV GLP-2 (11 nmol/ $\mathrm{kg} / \mathrm{d})$ or saline was initiated on day 2. Piglets were maintained for $14 \mathrm{~d}$. Clinical, functional, morphological, and histological outcomes were obtained.

RESULTS: JC-GLP-2 piglets had fewer days on PN (10.0 \pm 0.6 vs. $13.8 \pm 0.2)$, more days on EN ( $4.0 \pm 0.6$ vs. $0.2 \pm 0.2)$, a higher percentage of EN at termination ( $92 \pm 5$ vs. $52 \pm 10 \%$ ), fewer days of diarrhea $(8.0 \pm 0.7$ vs. $12.3 \pm 0.4)$, increased intestinal length ( $19 \pm 4$ vs. $-5 \pm 3 \%)$, and deeper jejunal crypts ( $248 \pm 21$ vs. $172 \pm 12 \mu \mathrm{m})$, compared with saline piglets.

CONCLUSION: GLP-2 therapy improves clinical, morphological, and histological outcomes of intestinal adaptation in a distal-intestinal resection model of SBS. Since this anatomical subtype represents the majority of clinical cases of neonatal SBS, these results support a potential role for GLP-2 therapy in pediatric SBS.

$S^{\mathrm{h}}$ hort bowel syndrome (SBS) results from massive resection of the intestine that leads to a shortened and functionally inadequate intestinal remnant for survival, growth, and hydration $(1,2)$. Parenteral nutrition (PN) is the mainstay of therapy for patients with SBS and, in children with SBS, PN is necessary for normal growth of the individual while the small intestinal remnant undergoes adaptation. The prolonged use of PN and long-term hospitalization are associated with frequent central venous catheter (CVC) complications, sepsis, cholestatic liver disease, liver failure, and failure to thrive $(1,3)$. The complications of SBS are significant, and account for more than 37\% of deaths in neonates with SBS (4). The significant morbidity and mortality associated with SBS, the considerable costs to society, and the poor quality of life for patients and their families warrant the need to wean patients off $\mathrm{PN}$ and to achieve enteral independence as quickly as possible $(2,5)$.

In the setting of SBS, there are several factors that influence successful adaptation of the remnant intestine. These factors include the length, anatomy, and function of the small and large intestinal remnant; the adaptive capacity of the intestinal remnant; the presence or absence of the ileocecal valve; the age of the patient; the initial diagnosis and disease burden; and exposure to enteral nutrients, pancreaticobiliary secretions (PBS), hormones, and growth factors (6). In this article, we have focused on the effects of remnant intestinal anatomy and exposure to exogenous glucagon-like peptide-2 (GLP-2) on small intestinal adaptation in neonatal piglet models of SBS.

GLP-2 is a 33-amino-acid peptide released from enteroendocrine L-cells of the distal ileum and colon following nutrient ingestion $(7,8)$. Clinical trials of human GLP-2 therapy for adult patients with SBS have demonstrated improvements in enteral energy absorption and fluid balances, decreased gastric emptying, decreased fecal output, and increased small intestinal villus height and crypt depth in patients treated with GLP-2 compared with placebo $(9,10)$. As a result, a long-acting analog of GLP-2 has recently been approved for use in adults with SBS (11). However, no trials on pediatric patients have been conducted to date.

We have previously studied the relationship between endogenous GLP-2 and intestinal adaptation in neonatal piglets as a translational model for the developing human (12). Neonatal piglets that undergo a distal-intestinal resection (JC) to 
completely remove the ileum have lower endogenous GLP-2 production, limited adaptation, and more severe intestinal failure compared with animals that undergo a mid-intestinal resection (JI) and have ileum present (12). This is relevant to the clinical situation in human infants as most causes of neonatal SBS are associated with congenital loss or resection of the ileum (1). Therefore, in this study, we hypothesized that exogenous administration of GLP-2 administration would improve clinical, functional, morphological, and histological outcomes of intestinal adaptation in a distal-intestinal resection neonatal piglet model of SBS. Since most early studies that investigated the effect of exogenous GLP-2 in animal models of SBS used mid-intestinal resection models, we also investigated the effect of exogenous GLP-2 administration on outcomes of intestinal adaptation in a mid-intestinal resection neonatal piglet model of SBS. Furthermore, to understand the mechanisms underlying any beneficial effects in these models, we determined the effects of exogenous GLP-2 on crypt cellular proliferation, villus apoptosis, and GLP-2 receptor mRNA expression.

\section{RESULTS}

\section{Plasma GLP-2 Levels}

Bioactive plasma GLP-2 levels were measured in the fed state for all animals on the day of surgery, when all animals achieved $50 \%$ nutrition by enteral feeds, and at termination (Figure 1). On the day of surgery, plasma GLP-2 levels were found to be in the range of $23.4 \pm 4$ to $30.7 \pm 6 \mathrm{pmol} / \mathrm{l}$ and did not differ between groups. At 50\% enteral nutrition (EN), endogenous GLP-2 levels were found to be 3.1 times higher in JI-saline animals compared with sham-saline animals (103.3 \pm 9.9 vs. $33.5 \pm 9.1 \mathrm{pmol} / 1, P<0.05)$, while there was no difference in levels between JC-saline and sham-saline animals $(68.7 \pm 11.1$ vs. $33.5 \pm 9.1 \mathrm{pmol} / \mathrm{l})$. Similarly, at termination, endogenous GLP-2 levels were 3.1 times higher in JI-saline animals as compared with sham-saline animals (161.0 $1 \pm 33.6$ vs. $52.6 \pm 6.9$ $\mathrm{pmol} / \mathrm{l}, P<0.05)$, while there remained no difference in levels between JC-saline and sham-saline animals (108.2 \pm 22.3 vs. $52.6 \pm 6.9 \mathrm{pmol} / \mathrm{l})$.

\section{Clinical Outcomes}

Clinical outcomes including the number of days on $\mathrm{PN}$, the number of days during which EN was the sole source of nutrition, the highest percentage of EN tolerated over the course of the trial, the percentage of EN at termination, and the number of days of diarrhea for the different surgical and treatment groups are summarized in Figure 2. For sham-GLP-2 as compared with sham-saline animals, there were no differences in any of the clinical outcomes. Over the study period, JI-GLP-2 animals achieved $17 \%$ more enteral feeds than JI-saline piglets ( $100 \pm 0$ vs. $83 \pm 7 \%, P<0.05$ ); however, there were no differences in the number of days on $\mathrm{PN}$, the number of days on $\mathrm{EN}$ as the sole nutrition source, the percentage of EN at termination, and the number of days of diarrhea between the two groups. In contrast, JC-GLP-2-treated piglets had 3.8 fewer days on $\mathrm{PN}(10.0 \pm 0.6 \mathrm{~d}$ vs. $13.8 \pm 0.2, P<0.05), 3.8$ more days on EN alone ( $4.0 \pm 0.6$ vs. $0.2 \pm 0.2 \mathrm{~d}, P<0.05)$, achieved $27 \%$
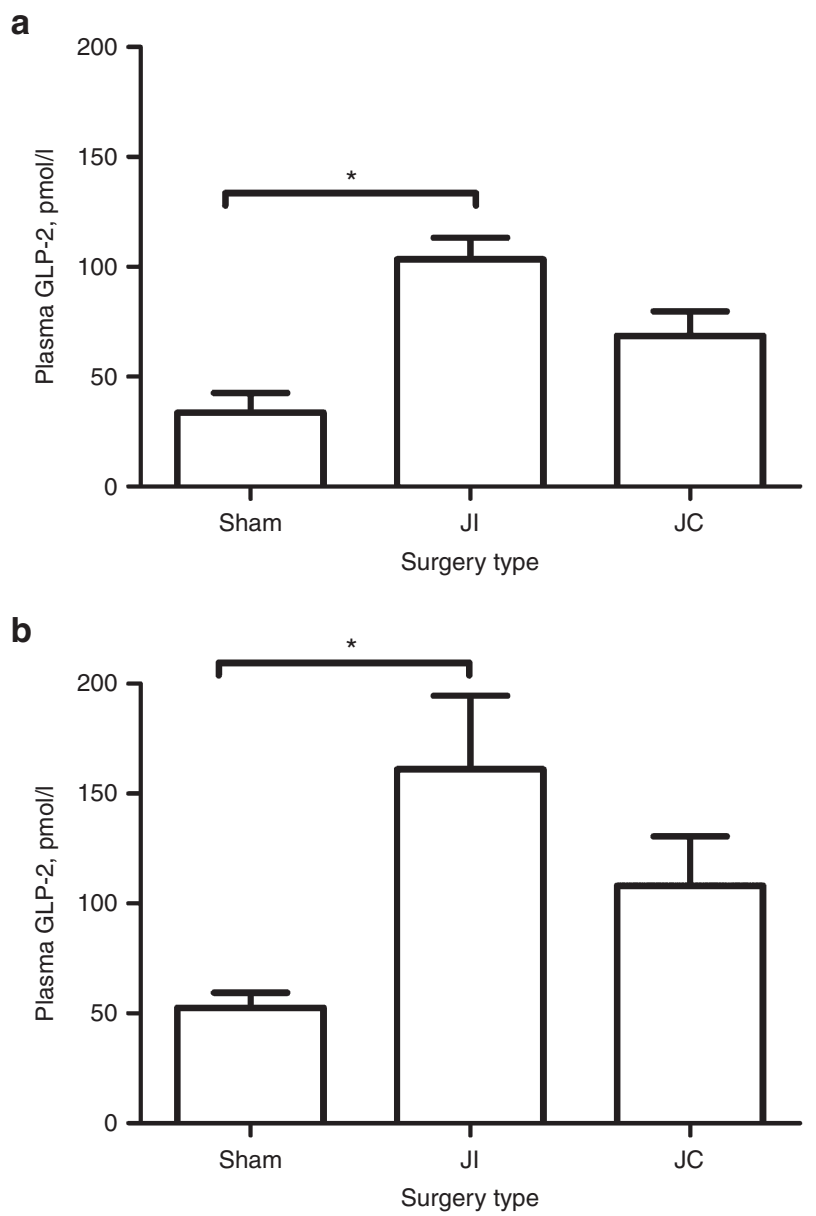

Figure 1. Plasma glucagon-like peptide-2 (GLP-2) concentrations for saline-treated sham, mid-intestinal resection (JI), and distal-intestinal resection (JC) groups at selected points in time. (a) $50 \%$ Enteral nutrition (EN) and (b) termination. ${ }^{*} P<0.05$ for comparison of means by one-way analysis of variance.

more EN over the study period ( $100 \pm 0$ vs. $73 \pm 7 \%, P<0.05$ ), tolerated $40 \%$ more EN at termination ( $92 \pm 5$ vs. $52 \pm 10 \%$, $P<0.05)$, and had 4.3 fewer days of diarrhea $(8.0 \pm 0.7$ vs. $12.3 \pm 0.4 \mathrm{~d}, P<0.05)$ compared with JC-saline controls.

\section{Enteral Fat Absorption}

No significant differences were observed in the absorption of enteral fat between the different surgical and treatment groups (Figure 3). Enteral fat absorption is not reported for sham animals because most of these animals did not produce feces during the fecal collection periods of the study.

\section{Intestinal Morphology}

Small intestinal length was measured during surgery (day 0) and at termination (day 14) for all animals. Absolute intestinal length at termination was $66 \mathrm{~cm}$ longer in JI-GLP-2 piglets ( $280 \pm 11$ vs. $214 \pm 6 \mathrm{~cm}, P<0.01)$ and $49 \mathrm{~cm}$ longer in JC-GLP-2 piglets $(204 \pm 12$ vs. $155 \pm 8 \mathrm{~cm}, P<0.01)$ compared with the respective saline-treated piglets. The percent change in small intestinal length from surgery to termination was 39\% higher for JI-GLP-2 piglets $(71 \pm 8$ vs. $32 \pm 2 \%, P=0.001)$ and $24 \%$ higher 
a

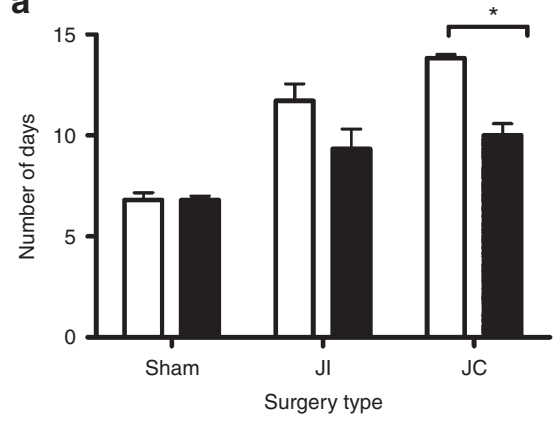

b

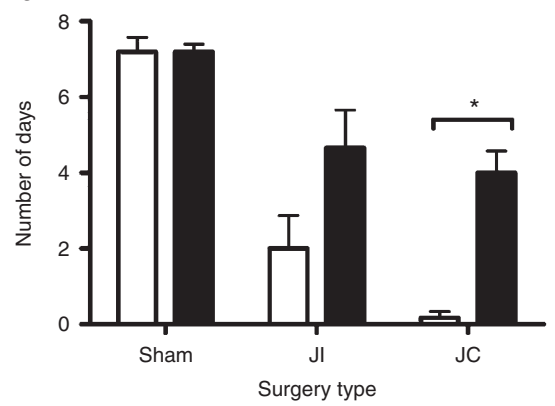

C

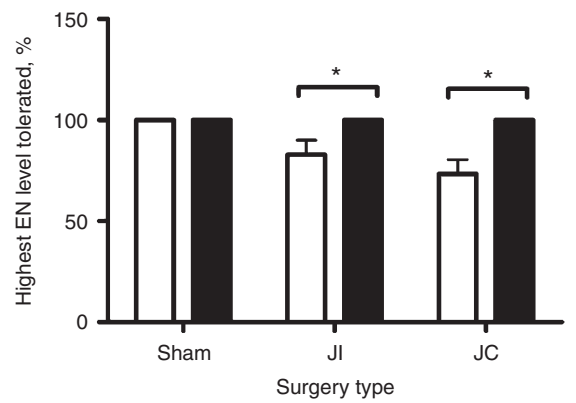

d

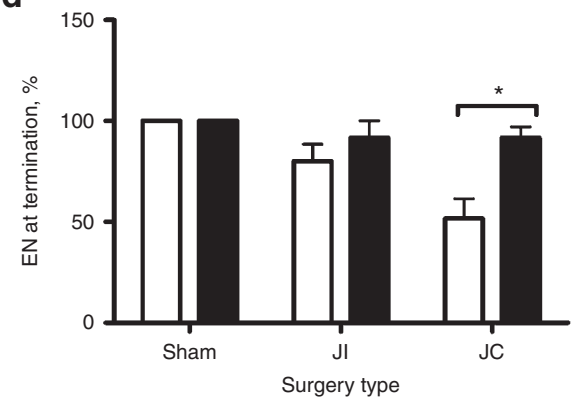

e

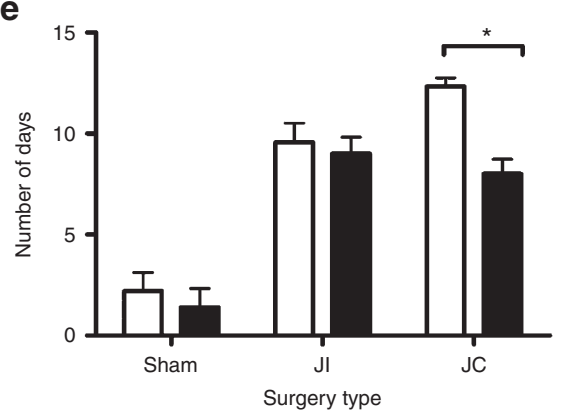

Figure 2. Clinical outcomes relating to parenteral nutrition (PN) and enteral nutrition (EN) for sham, mid-intestinal resection (JI), and distal-intestinal resection (JC) groups. (a) Number of days on PN, (b) number of days on EN as the sole nutrition source, (c) highest percentage of EN tolerated during trial, (d) percentage of EN at termination, and (e) number of days of diarrhea. White bars indicate saline treatment and black bars indicate glucagon-like peptide-2 (GLP-2) treatment. Comparison of means by two-way analysis of variance. ${ }^{*} P<0.05$ for subgroup comparison of means by Student's $t$-test.

in JC-GLP- 2 piglets ( $19 \pm 4$ vs. $-5 \pm 3 \%, P=0.001)$ relative to the respective saline-treated animals. In contrast, although surgery was a significant factor affecting small intestinal weight per piglet body weight, with sham and JI animals having higher values than JC animals, GLP-2 therapy had no effect on small intestinal weight per piglet body weight for any of the surgical groups.

Jejunal villi were 1.4 times higher in JI-GLP-2 animals compared with JI-saline piglets $(1,006 \pm 92$ vs. $741 \pm 37 \mu \mathrm{m}, P<$ $0.05)$. There were no differences in jejunal villus height between sham-saline and sham-GLP-2 animals, or between JC-salineand JC-GLP-2-treated piglets. Relative to saline-treated piglets, jejunal crypts were 1.3 times deeper in GLP-2-treated JI animals $(234 \pm 9$ vs. $177 \pm 14 \mu \mathrm{m}, P<0.05)$ and 1.4 times deeper in GLP-2-treated JC animals $(248 \pm 21$ vs. $172 \pm 12 \mu \mathrm{m}, P<$ $0.05)$. There were no differences in jejunal crypt depth between sham-saline- and sham-GLP-2-treated animals (Figure 4). In the ileum of sham and JI animals, there were no differences in either villus height or crypt depth between saline- and GLP-2treated groups (data not shown).

There were no significant differences in the total number of cells with jejunal Ki67 immunoreactivity in any of the crypt zones between the different surgical and treatment groups, and there were no statistically significant differences in absolute jejunal villus cleaved caspase-3 immunoreactivity for the different surgical and treatment groups (data not shown). However, relative jejunal villus cleaved caspase- 3 immunoreactivity was 1.1- and 1.2-fold lower for JI-GLP-2 and JC-GLP-2 animals, respectively, compared with sham-GLP-2 animals $(1.2 \pm 0.2$ and $1.1 \pm 0.1$ vs. $2.3 \pm 0.6$ fold of control, $P<0.05)$.

\section{GLP-2 Receptor mRNA Expression in Jejunal, Ileal, and Colonic} Tissue

At the end of the study, jejunal GLP-2R mRNA expression was 12.5 times higher in JI-GLP-2 animals compared with JI-saline piglets $(2.5 \pm 1.1$ vs. $0.2 \pm 0.1, P<0.05)$. There were no differences in jejunal GLP-2R mRNA expression between GLP-2- and saline-treated animals for the sham and JC groups. In the ileum, GLP-2R mRNA expression was similarly 13.9 times higher in the sham-GLP-2 group compared with the sham-saline group $(11.1 \pm 5.7$ vs. $0.8 \pm 0.5, P<0.01)$ and 12 times higher in the JI-GLP-2 group compared with the JI-saline group $(2.4 \pm 0.4$ and $0.2 \pm 0.1, P<0.001)$. Finally, in the colon, GLP-2R mRNA expression was 12.7 times higher in the JI-GLP-2 group compared with the JI-saline group (7.6 \pm 3.1 vs. $0.6 \pm 0.1, P<0.05)$ and 15.4 times higher in JC-GLP-2 piglets compared with JC-saline piglets $(18.5 \pm 7.9$ and $1.2 \pm 0.5, P<0.05)$.

\section{DISCUSSION}

We have previously shown that endogenous bioactive GLP-2 levels are reduced in a distal-intestinal resection neonatal piglet model of SBS that lacks remnant ileum compared with a mid-intestinal resection neonatal piglet model of SBS in which the ileum is present, and that former animals have blunted clinical and morphological outcomes of intestinal adaptation compared with the latter (12). Based on these findings, we hypothesized that exogenous administration of human GLP-2 would improve clinical, functional, morphological, and histological outcomes of intestinal adaptation in a distal-intestinal 
resection neonatal piglet model of SBS that lacks remnant ileum.

This study is the first to report the effects of human GLP-2 therapy on clinically relevant outcomes of intestinal adaptation, including PN requirements, EN intake, body weight changes, and the number of days of diarrhea, in a neonatal model of SBS. We have demonstrated here that exogenous human GLP-2 administration augments clinical outcomes of intestinal adaptation in a distal-intestinal resection neonatal piglet model of SBS. Hence, JC-GLP-2-treated piglets had fewer days on $\mathrm{PN}$, more days on EN alone, a higher percentage of EN tolerated, a higher percentage of EN at the end of the trial period, and fewer days of diarrhea compared with JC-saline-treated piglets. While JI-GLP-2-treated animals tolerated a higher percentage of enteral feeds than JI-saline-treated piglets, no differences in all the other clinical outcomes were observed.

Since JC-GLP-2-treated piglets had fewer days of diarrhea and better enteral tolerance relative to JC-saline-treated animals, we also expected that nutrient absorption and measures of functional adaptation would be improved in these animals. However, no differences in enteral fat absorption between GLP-2-treated and saline-treated animals were observed in each respective surgical group. The effect of GLP-2 administration on enteral fat absorption in animal models of SBS has not been previously studied, and it might therefore be the case that microscopic structural adaptation or other unmeasured parameters of functional adaptation are occurring.

In patients with SBS, a strong predictor of clinical success and weaning from PN is remnant intestinal length (13-18). Hence, it was expected that the groups of animals that had better clinical outcomes would also have longer small intestines at the end of the trial period. JC-GLP-2-treated animals had better clinical outcomes than JC-saline-treated piglets, and both absolute small intestinal length and the percent change in intestinal length were higher in the JC-GLP-2-treated group compared with the JC-saline group. Interestingly, even though both absolute small intestinal length and the percent change in small intestinal length were higher for JI-GLP-2-treated animals relative to JI-saline-treated piglets, no differences in clinical outcomes between these two groups were observed. The absence of better clinical outcomes in JI-GLP-2-treated animals as compared with JI-saline-treated piglets, despite an increase in absolute and relative small intestinal length, may suggest that other forms of intestinal adaption (e.g., microscopic structural adaptation and functional adaptation) are occurring in piglets with remnant ileum, regardless of exogenous GLP-2 administration.

To further explain the observed differences in clinical outcomes between JC-saline- and JC-GLP-2-treated animals, it was also expected that similar differences in microscopic intestinal architecture would be observed between the two groups. In the jejunum, crypts were found to be significantly deeper in JC- and JI-GLP-2-treated animals relative to the respective saline controls, and jejunal villi were found to be significantly longer for JI-GLP-2-treated animals relative to JI-saline-treated piglets. Even though the results were not
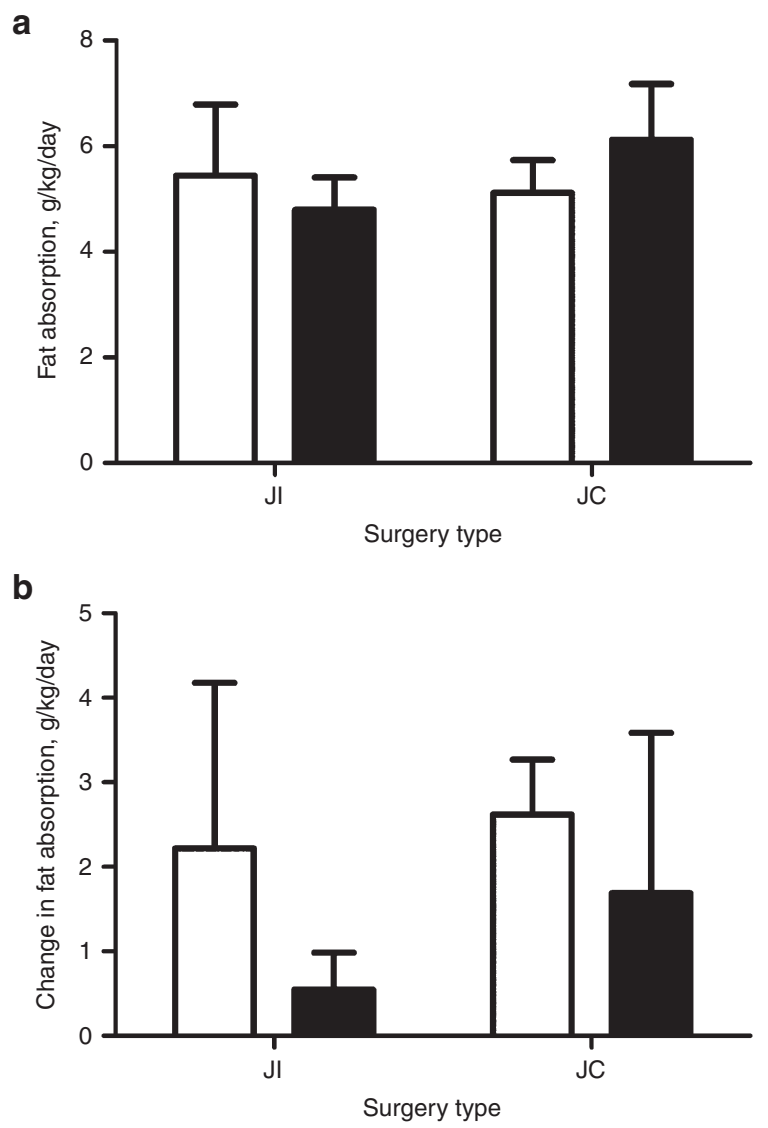

Figure 3. Enteral fat absorption for mid-intestinal resection (JI) and distalintestinal resection (JC) groups. (a) Days 12-14 and (b) change from days 5-7 and days 12-14. White bars indicate saline treatment and black bars indicate glucagon-like peptide-2 (GLP-2) treatment. Comparison of means by two-way analysis of variance.

statistically significant, there was a trend towards longer jejunal villi for both JC- and sham-GLP-2-treated animals relative to the respective treatment controls. Hence, evidence for the presence of microscopic intestinal hyperplasia and adaptation in the jejunum of surgically resected GLP-2-treated neonatal piglets, without ileum, was observed.

Intestinal cellular proliferation and apoptosis were also evaluated to delineate the mechanisms underlying the observed differences in intestinal villus length and crypt depth between saline- and GLP-2-treated surgical groups. In groups with longer villi and/or deeper crypts, an increase in crypt cellular proliferation and/or a decrease in villus apoptosis was expected. Even though jejunal villus height and/or crypt depth in GLP2-treated JI and JC piglets relative to saline-treated animals in the same surgical groups were increased, no differences in villus apoptosis or crypt cellular proliferation were observed. Pereira-Frantini et al. (19) found both an increase in enterocyte proliferation and apoptosis, and a decrease in ileal villus height and crypt depth with GLP-2 administration in a midintestinal resection juvenile piglet model of SBS. In the latter study, the resulting decrease in ileal villus height and crypt depth with GLP-2 therapy may have resulted from a greater extent of enterocyte apoptosis relative to proliferation. In this 

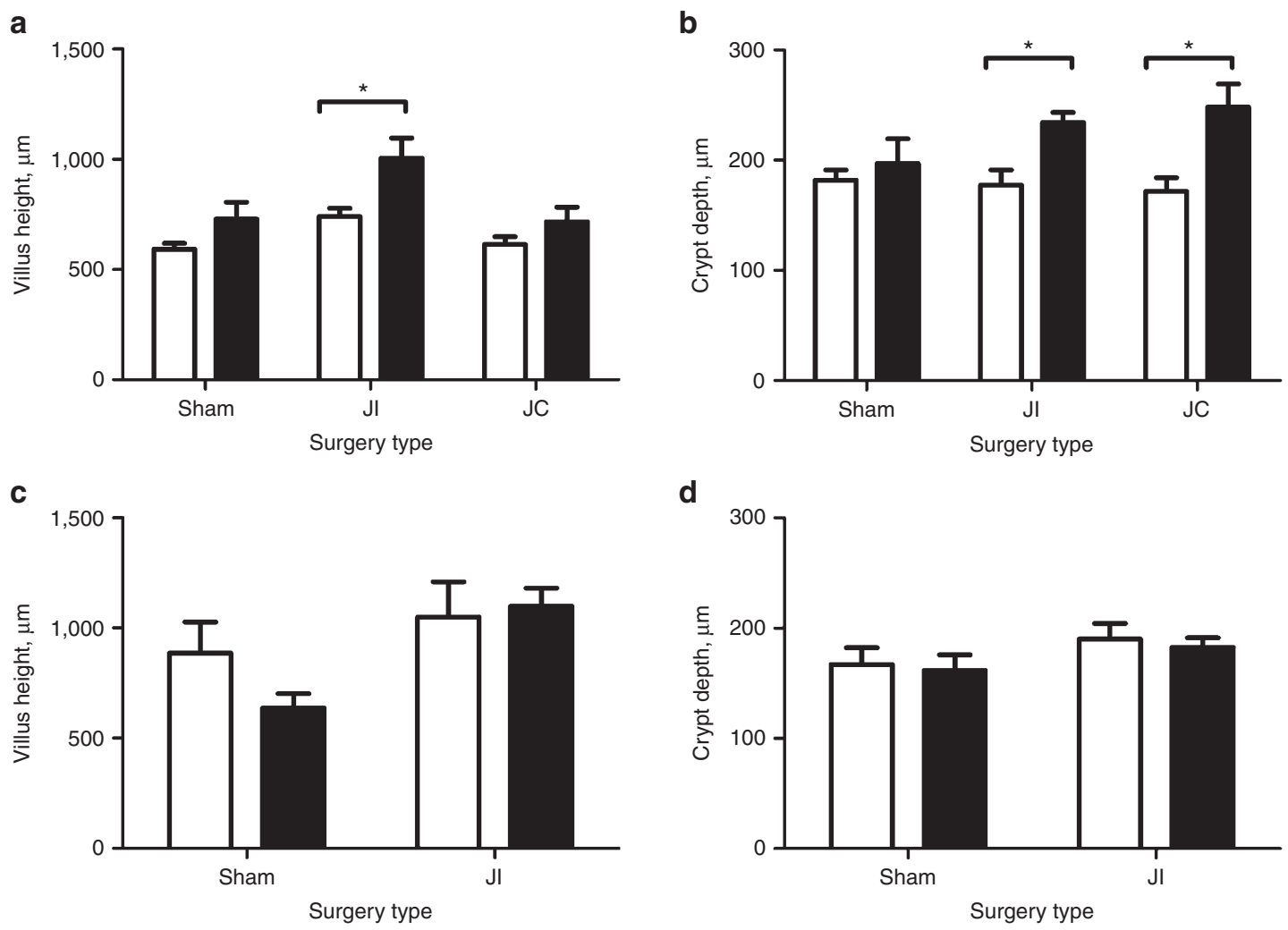

Figure 4. Small intestinal mucosal morphometry for sham, mid-intestinal resection (JI), and distal-intestinal resection (JC) groups. (a) Jejunal villus height, (b) jejunal crypt depth, (c) ileal villus height, and (d) ileal crypt depth. White bars indicate saline treatment and black bars indicate glucagon-like peptide-2 (GLP-2) treatment. Comparison of means by two-way analysis of variance. ${ }^{*} P<0.05$ for subgroup comparison of means by Student's $t$-test.

study, absolute changes in cellular activity do not appear to provide an explanation for the observed changes in microscopic intestinal architecture. However, when cleaved caspase-3 immunoreactivity for GLP-2-treated piglets relative to saline-treated piglets in the same surgical group was evaluated, JI- and JC-treated animals had lower fold of control cleaved caspase-3 positive cells than sham animals. That is, relative crypt cellular apoptosis in GLP-2-treated animals compared with saline-treated animals within the same surgical group was lower in JI and JC piglets as compared with sham piglets. This finding provides some support to the results of our study.

Enterocyte cellular hyperplasia in the setting of exogenous GLP-2 administration has been extensively studied in rodents with intact intestinal anatomy, and both an increase in epithelial cell proliferation and a decrease in apoptosis are observed with GLP-2 administration (20-23). In a mid-intestinal resection model of SBS, Martin et al. (24) demonstrated that GLP-2 therapy was associated with an increase in ileal cell proliferation with no change in ileal enterocyte apoptosis, and in a distal-intestinal resection model of SBS, Sigalet et al. (25) found an increase in both jejunal cellular proliferation and apoptosis with GLP-2 administration. It is plausible that the proliferative and apoptotic activity of piglet intestinal epithelial cells changes with intestinal development over the course of the neonatal period-hence, if enterocyte proliferation in neonatal piglets declines over time, while apoptosis increases, the time at which the intestinal tissue is sampled will impact the results of enterocyte proliferative and apoptotic frequency. Burrin et al. (26) demonstrated that GLP-2 administration was associated with an increase in intestinal epithelial cell proliferation and a decrease in apoptosis. Piglets in this study were terminated after $7 \mathrm{~d}$ for collection of intestinal specimens, while piglets in the present study were maintained for $14 \mathrm{~d}$. If intestinal samples were obtained at an earlier time point during the trial period in the present study, different results with regard to crypt cellular apoptosis and proliferation may thus have been observed.

Understanding the mechanism of action of GLP-2 has been challenging. This is in part due to the multiple mediators involved in the actions of GLP-2 and the complexity of GLP-2R expression. The tropic effects of GLP-2 on intestinal epithelium are likely exerted indirectly via downstream growth factors, since GLP-2R is not identified on intestinal epithelial cells (27). Currently, there are few studies that have examined the effects of exogenous GLP-2 administration on GLP-2R expression. Dubé et al. (28) demonstrated no differences in distal jejunal GLP-2R mRNA expression between mice administered exogenous human $\left(\mathrm{Gly}^{2}\right) \mathrm{GLP}-2$ or saline, while de Heuvel et al. (29) found an increase in GLP-2R mRNA expression in rodent colonic submucosal enteric neurons exposed to human GLP-2 in vitro. In our study, GLP-2R mRNA expression was found to be higher in jejunal, ileal, and colonic tissue for JI piglets treated with GLP-2 as compared with saline-treated animals. Ileal GLP-2R mRNA expression 
was also higher in sham-GLP-2-treated animals relative to sham-saline animals, and although jejunal and colonic GLP-2R mRNA expression was not significantly different between sham-GLP-2- and sham-saline-treated animals, there was a trend towards higher expression in the former group. Colonic GLP-2R mRNA expression was higher in JC-GLP-2-treated animals relative to JC-saline animals and, again, although jejunal GLP-2R mRNA expression was not significantly different between JC-GLP-2- and JC-saline-treated animals, there was a trend towards higher expression in the former group. These observations suggest that exogenous GLP-2 administration increases GLP-2R mRNA transcripts in all intestinal anatomical regions of JI neonatal piglets (jejunum, ileum, and colon), and increases GLP-2R mRNA expression in some regions of sham (ileum) and JC piglets (colon). Hence, differences in GLP-2R expression may not be responsible for the differences in outcomes of intestinal adaptation observed between JI- and JC-GLP-2-treated neonatal piglets, and there may be a downstream mediator of GLP-2, such as insulin-like growth factor-1 (27), that is more prominently expressed in JC-GLP-2-treated neonatal piglets. This hypothesis is worthy of investigation in future studies.

\section{Conclusions}

In our study, exogenous human GLP-2 improved the morphological and histological outcomes of intestinal adaptation in mid-intestinal resection and distal-intestinal resection neonatal piglet models of SBS. However, improved clinical outcomes were only observed with GLP-2 administration in the distalintestinal resection neonatal piglet model. Since the distalintestinal resection neonatal piglet model of SBS replicates the most common remnant anatomy in neonatal SBS, these preliminary results support a role for GLP-2 therapy to treat pediatric SBS patients who have undergone a distal-intestinal resection and lack remnant ileum.

\section{METHODS}

\section{Research Ethics Approval}

All procedures in this study were approved by the University of Alberta Animal Care and Use Committee for Livestock (\#125/02/11).

\section{Animals and Surgical Procedures}

Fifty-one male Large/Landrace White Cross piglets (Hypor, Regina, SK, Canada) of age $2-5 \mathrm{~d}$ and weighing $1.8-2.6 \mathrm{~kg}$ underwent general anesthesia, and received a 5-French CVC in the left external jugular vein and a 10-French gastrostomy tube in accordance with procedures as reported previously $(30,31)$. A laparotomy was performed and small intestinal length was measured from the ligament of Treitz (LOT) to the ileocecal junction. In the surgical control or sham group, no intestinal resection was performed. In the JI group, piglets underwent a $75 \%$ mid-intestinal resection with a jejunoileal anastomosis. In the JC group, piglets underwent a $75 \%$ distal-intestinal resection with a jejunocolic anastomosis.

Postoperatively, piglets were housed in individual Plexiglas cages to which they were secured by a tether-swivel system (Alice King Chatham Medical Arts, Los Angeles, CA). Catheters were channeled through the internal lumen of the swivel and connected to a dual-channel infusion pump (IVAC Signature Gold Infusion Pump; ALARIS Medical Systems, San Diego, CA). The temperature of the room was maintained at $25^{\circ} \mathrm{C}$ with the aid of a heat lamp, and lighting was established with a 12-h light/dark cycle.

\section{Nutrition}

Immediately after surgery, elemental PN was initiated by infusion through the CVC. The amino acid content of the PN solution was similar to that used by Wykes et al. (30), and was based on human milk protein (Vaminolact; Fresenius Kabi, Bad Homburg, Germany). The targeted energy intake was $1,100 \mathrm{~kJ} /(\mathrm{kg} \mathrm{d})$, with amino acids providing $27 \%$, carbohydrate $37 \%$, and fat $36 \%$ of energy. Target nutrient intake included $18.0 \mathrm{~g}$ amino acids/(kg d), $29.0 \mathrm{~g}$ glucose/(kg d), and $10.4 \mathrm{~g} \mathrm{fat} /(\mathrm{kg} \mathrm{d})$, and was estimated from daily nutritional requirements for sow-fed piglets (30).

On postoperative day 2, EN was introduced via the gastric catheter. The EN solution had a similar amino acid, lipid, and mineral content to the PN solution; however, glucose was substituted with glucose polymer module (Polycose; Abbot Nutrition Canada, Saint-Laurent, Québec, Canada) in order to reduce the osmolarity of the solution. The EN solution was isocaloric and isonitrogenous to the PN solution. EN started at $10 \%$ of the total nutritional fluid rate $(1.3 \mathrm{ml} /$ ( $\mathrm{kg} \mathrm{h}$ ). In the first week, if the piglet gained $50 \mathrm{~g} / \mathrm{d}$ and was neither vomiting nor dehydrated, EN was increased by $10 \%$ every $24 \mathrm{~h}$. In the second week, if the piglet gained $100 \mathrm{~g} / \mathrm{d}$ and was neither vomiting nor dehydrated, EN was increased by $10 \%$ every $24 \mathrm{~h}$. These parameters for weight gain and associated feeding of surgical short bowel piglets have been previously validated in this laboratory (31). If stool output was formed or absent, EN was increased by $10 \%$ every $12 \mathrm{~h}$. With each increase in the rate of EN by $10 \%$, the rate of PN was proportionally decreased in order to maintain a total nutritional intake of $324 \mathrm{ml} /(\mathrm{kg} . \mathrm{d})(13.5 \mathrm{ml} /(\mathrm{kg} \mathrm{h})$.

\section{Glucagon-Like Peptide-2 or Saline Infusion}

On postoperative day 2, IV human GLP-2 (Human GLP-2(1-33); CS9065; Lot I074 with $96.83 \%$ purity confirmed by HPLC; CS Bio, Menlo Park, CA) $(11 \mathrm{nmol} /(\mathrm{kg} \mathrm{d})$ at a rate of $0.42 \mathrm{ml} /(\mathrm{kg} \mathrm{h})$ was administered continuously through the jugular venous catheter by a syringe pump (NE-300 Just Infusion Syringe Pump; New Era Pump Systems, Farmingdale, NY) to all piglets randomized to the GLP-2 treatment group (i.e., sham-GLP-2, JI-GLP-2, and JC-GLP-2). Piglets randomized to the vehicle group (i.e., sham-saline, JI-saline, and JC-saline) received normal saline ( $0.9 \%$ sodium chlorine; Baxter, Mississauga, ON, Canada).

\section{Plasma Glucagon-Like Peptide-2 Collection and Radioimmunoassay}

Whole blood was collected in the fed state at baseline (after insertion of the CVC but prior to laparotomy), when EN constituted $50 \%$ of total nutrition, and prior to termination. Plasma was obtained by centrifuging the blood sample at 2,500 rpm for $10 \mathrm{~min}$. The supernatant was collected and several aliquots of plasma were stored at $-80^{\circ} \mathrm{C}$. Plasma bioactive GLP-2 was determined by radioimmunoassay with the use of an antibody specific to the N-terminus of GLP-2 as previously reported $(32,33)$.

\section{Fecal Sampling, Enteral Fat Absorption, and Functional Outcomes}

Functional intestinal adaptation was assessed by measuring enteral fat absorption. Fecal matter was collected for a 24 - to 48 -h period starting on day 5 and day 12 of the trial. Feces were collected in drainable ostomy pouches (Two-Piece Pouch System; Hollister, Aurora, ON, Canada) placed over the perianal region. After 6-8h during each fecal collection period, feces were collected and weighed. To determine the amount of enteral fat delivered to piglets, EN bags were weighed at the start and end of each 6- to 8-h period. If leakage of feces was suspected during the 6- to 8-h collection period, these samples were discarded from the analysis. Feces were freeze-dried for $6 \mathrm{~d}$ prior to performing the fat-content analysis. Each fecal collection was analyzed in duplicate with samples weighing $1-2 \mathrm{~g}$ each. Over a 6 -h period, fat was extracted from each sample by petroleum ether distillation using the Goldfisch apparatus (method Aa 4-38, AOAC 2000), and the fat content of each sample was determined by the mass of the extract. Fat absorption $(\mathrm{g} /(\mathrm{kg}))$ was calculated by subtracting the total fat content of all the feces obtained over the collection period from the total amount of lipid delivered during the same collection period. 


\section{Terminal Laparotomy, Tissue Specimens, and Morphological Outcomes}

At the end of the study (day 14), piglets underwent a laparotomy under general anesthetic. Intestinal length was measured from the LOT to the terminal ileum in sham and JI surgical groups, and from the LOT to the jejunocolic anastomosis in the JC group. A $2 \mathrm{~cm}$ specimen of intestinal tissue was obtained at $60 \mathrm{~cm}$ distal to the LOT (jejunum), $20 \mathrm{~cm}$ proximal to the ileoceceal valve when applicable (terminal ileum), and $5 \mathrm{~cm}$ distal to the ileocecal valve or jejunocolic anastomosis (colon). All tissue specimens were preserved in a 10\% buffered formaldehyde solution (Histoprep; Fisher Scientific, Ottawa, ON, Canada) for histology. The entire small intestine was subsequently detached from the mesentery, cleared of intestinal contents, and weighed. Morphological outcomes included small intestinal length and weight.

\section{Histology}

Histological specimens were prepared and analyzed by a certified veterinary pathologist (P.N.N.) who was blinded to the treatment arms of the study. Two to three millimeter cross-sections of intestinal tissue were obtained from the specimens preserved in formaldehyde. These were embedded in paraffin for preparation of $5 \mu \mathrm{m}$ sections according to standard techniques. Ten well-oriented villi and crypts were measured on 2-3 hematoxylin and eosin (H\&E)-stained sections per piglet.

\section{Immunohistochemistry}

Intestinal sections ( $4 \mu \mathrm{m}$ ) were stained for Ki-67 (a marker of cellular proliferation (34)) and cleaved caspase-3 (a marker of cellular apoptosis (35)) at Prairie Diagnostic Services (Saskatoon, SK, Canada) using a commercial staining platform (Benchmark Staining Platform; Ventana Medical Systems, Tucson, AZ) with a streptavidin-biotin detection system (BMK iVIEW DAB Paraffin detection kit; Ventana Medical Systems,). The manufacturer's protocol was followed for each antibody, with the Ki-67 (mouse monoclonal to Ki-67 clone MIB-1 (M7240), Dako Canada, Mississauga, ON, Canada) and cleaved caspase-3 (rabbit polyclonal to cleaved caspase-3, Asp175 (9661), Cell Signaling Technology, Danvers, MA) antibodies used at dilution of $1: 100$. For each staining run, sections of normal intestine were included to ensure consistency of positive staining, and primary antibody was omitted as a control for each section.

For each intestinal specimen, 20 well-oriented crypts were identified from an average of $2-3$ slides. Along the right half of each crypt, cell positions were identified according to the methods reported by Potten and Veradi et al. (36-38), with the cell located at the base of the crypt designated as cell-position 1 . Cells on the right column of each half-crypt were divided into eight zones ( 3 cells/zone) up to the 24 th position. Ki-67 expression was quantified by determining the percentage of positively stained cells per half-crypt zone. Cellular apoptosis was assessed using 20 well-oriented villi from an average of $2-3$ slides. Cells that positively stained for active caspase- 3 were identified and expressed as a percentage of the first 60 cells from the villus tip (30 on each side (39)). All slides were scored by the same pathologist (P.N.N.) blinded to treatment.

\section{Quantitative Real-Time Polymerase Chain Reaction (qRT-PCR): GLP-2 Receptor mRNA Quantification}

Total mRNA was extracted from samples of jejunum, ileum, and colon at baseline and termination using the TRIzol reagent (Invitrogen Life Technologies, Burlington, ON, Canada), as previously described (40). For each sample, $1 \mu \mathrm{g}$ of mRNA was reverse transcribed with Superscript II (Invitrogen Life Technologies) and random hexamer primers (Amersham, Arlington Heights, IL). Two-step qRT-PCR analysis was performed using Express SYBR GreenER (Invitrogen Life Technologies) and porcine-specific primers. For each sample, DNA amplification was performed in duplicate with the 7900HT Fast Real-Time PCR System (Applied Biosystems, Foster City, CA). Data analysis was performed using the 7900HT Fast Real-Time PCR System software (SDS 2.3; Applied Biosystems). Relative GLP-2R mRNA quantification was determined using the $\Delta \Delta \mathrm{C}_{(\mathrm{t})}$ method with ribosomal $18 \mathrm{~S}$ as the internal control (28).
GLP-2R mRNA expression in GLP-2-treated animals is expressed as a fold increase over vehicle-treated animals within the same surgical group.

\section{Statistical Analysis}

Experimental data are expressed as mean \pm SE. Some data are expressed as counts. Data expressed as means were compared by two-way ANOVA for a $2 \times 2$ factorial design of surgery and treatment factors followed by Student's $t$-test or one-way ANOVA with Tukey's post hoc analysis, as appropriate. $P$ values $<0.05$ were considered to be statistically significant. All statistical analyses were performed using GraphPad Prism 5.03 for Windows (GraphPad Software, San Diego, CA).

\section{ACKNOWLEDGMENTS}

We acknowledge and thank Laurie Wallace, Elaine de Heuvel, Estrella Bulloch, Zheng Hua, Christine Pendlebury, Rhian Ormond, Charlane Gorsak, Joan Turchinsky, Bolette Hartmann, Jens Holst, Benjamin Alman, and Johane Allard for their contributions to this research.

\section{STATEMENT OF FINANCIAL SUPPORT}

This work was supported by grants from the SickKids Foundation, Institute of Human Development, Child and Youth Health, Canadian Institutes of Health Research (IHDCYH-CIHR) National Grants Program (XG08-030R), Canadian Institutes of Health Research Master's Award: Frederick Banting \& Charles Best Canada Graduate Scholarship, the Molly Towell Perinatal Research Foundation, and the Women's and Children's Health Research Institute of Alberta.

Disclosure: The authors declare no conflict of interest.

\section{REFERENCES}

1. Wales PW, Christison-Lagay ER. Short bowel syndrome: epidemiology and etiology. Semin Pediatr Surg 2010;19:3-9.

2. Thompson JS, Rochling FA, Weseman RA, Mercer DF. Current management of short bowel syndrome. Curr Probl Surg 2012;49:52-115.

3. Sigalet DL. Short bowel syndrome in infants and children: an overview. Semin Pediatr Surg 2001;10:49-55.

4. Wales PW, de Silva N, Kim J, Lecce L, To T, Moore A. Neonatal short bowel syndrome: population-based estimates of incidence and mortality rates. J Pediatr Surg 2004;39:690-5.

5. Spencer AU, Kovacevich D, McKinney-Barnett M, et al. Pediatric short-bowel syndrome: the cost of comprehensive care. Am J Clin Nutr 2008;88:1552-9.

6. Cisler JJ, Buchman AL. Intestinal adaptation in short bowel syndrome. J Investig Med 2005;53:402-13.

7. Brubaker PL, Drucker DJ. Minireview: Glucagon-like peptides regulate cell proliferation and apoptosis in the pancreas, gut, and central nervous system. Endocrinology 2004;145:2653-9.

8. Brubaker PL. A beautiful cell (or two or three?). Endocrinology 2012;153:2945-8.

9. Jeppesen PB, Hartmann B, Thulesen J, et al. Glucagon-like peptide 2 improves nutrient absorption and nutritional status in short-bowel patients with no colon. Gastroenterology 2001;120:806-15.

10. Jeppesen PB, Sanguinetti EL, Buchman A, et al. Teduglutide (ALX-0600), a dipeptidyl peptidase IV resistant glucagon-like peptide 2 analogue, improves intestinal function in short bowel syndrome patients. Gut 2005;54:1224-31.

11. Jeppesen PB. Teduglutide for the treatment of short bowel syndrome. Drugs Today 2013;49:599-614.

12. Hua Z, Turner JM, Sigalet DL, et al. Role of glucagon-like peptide-2 deficiency in neonatal short-bowel syndrome using neonatal piglets. Pediatr Res 2013;73:742-9.

13. Andorsky DJ, Lund DP, Lillehei CW, et al. Nutritional and other postoperative management of neonates with short bowel syndrome correlates with clinical outcomes. J Pediatr 2001;139:27-33.

14. Quirós-Tejeira RE, Ament ME, Reyen L, et al. Long-term parenteral nutritional support and intestinal adaptation in children with short bowel syndrome: a 25-year experience. J Pediatr 2004;145:157-63. 
15. Sondheimer JM, Cadnapaphornchai M, Sontag M, Zerbe GO. Predicting the duration of dependence on parenteral nutrition after neonatal intestinal resection. J Pediatr 1998;132:80-4.

16. Spencer AU, Neaga A, West B, et al. Pediatric short bowel syndrome: redefining predictors of success. Ann Surg 2005;242:403-9; discussion 409-12.

17. Wales PW, de Silva N, Kim JH, Lecce L, Sandhu A, Moore AM. Neonatal short bowel syndrome: a cohort study. J Pediatr Surg 2005;40:755-62.

18. Weaver LT, Austin S, Cole TJ. Small intestinal length: a factor essential for gut adaptation. Gut 1991;32:1321-3.

19. Pereira-Fantini PM, Nagy ES, Thomas SL, et al. GLP-2 administration results in increased proliferation but paradoxically an adverse outcome in a juvenile piglet model of short bowel syndrome. J Pediatr Gastroenterol Nutr 2008;46:20-8.

20. Drucker DJ, Erlich P, Asa SL, Brubaker PL. Induction of intestinal epithelial proliferation by glucagon-like peptide 2. Proc Natl Acad Sci USA 1996;93:7911-6.

21. Tsai CH, Hill M, Asa SL, Brubaker PL, Drucker DJ. Intestinal growthpromoting properties of glucagon-like peptide-2 in mice. Am J Physiol 1997;273(1 Pt 1):E77-84.

22. Tsai CH, Hill M, Drucker DJ. Biological determinants of intestinotrophic properties of GLP-2 in vivo. Am J Physiol 1997;272(3 Pt 1):G662-8.

23. Ghatei MA, Goodlad RA, Taheri S, et al. Proglucagon-derived peptides in intestinal epithelial proliferation: glucagon-like peptide-2 is a major mediator of intestinal epithelial proliferation in rats. Dig Dis Sci 2001;46:1255-63.

24. Martin GR, Wallace LE, Sigalet DL. Glucagon-like peptide-2 induces intestinal adaptation in parenterally fed rats with short bowel syndrome. Am J Physiol Gastrointest Liver Physiol 2004;286:G964-72.

25. Sigalet DL, Bawazir O, Martin GR, et al. Glucagon-like peptide-2 induces a specific pattern of adaptation in remnant jejunum. Dig Dis Sci 2006;51:1557-66.

26. Burrin DG, Stoll B, Guan X, Cui L, Chang X, Holst JJ. Glucagon-like peptide 2 dose-dependently activates intestinal cell survival and proliferation in neonatal piglets. Endocrinology 2005;146:22-32.

27. Dubé PE, Brubaker PL. Frontiers in glucagon-like peptide-2: multiple actions, multiple mediators. Am J Physiol Endocrinol Metab 2007;293:E460-5.

28. Dubé PE, Forse CL, Bahrami J, Brubaker PL. The essential role of insulinlike growth factor-1 in the intestinal tropic effects of glucagon-like peptide-2 in mice. Gastroenterology 2006;131:589-605.
29. de Heuvel E, Wallace L, Sharkey KA, Sigalet DL. Glucagon-like peptide 2 induces vasoactive intestinal polypeptide expression in enteric neurons via phophatidylinositol 3-kinase-? signaling. Am J Physiol Endocrinol Metab 2012;303:E994-1005.

30. Wykes LJ, Ball RO, Pencharz PB. Development and validation of a total parenteral nutrition model in the neonatal piglet. J Nutr 1993;123:124859.

31. Turner JM, Wales PW, Nation PN, et al. Novel neonatal piglet models of surgical short bowel syndrome with intestinal failure. J Pediatr Gastroenterol Nutr 2011;52:9-16.

32. Hartmann B, Johnsen AH, Orskov C, Adelhorst K, Thim L, Holst JJ. Structure, measurement, and secretion of human glucagon-like peptide-2. Peptides 2000;21:73-80.

33. Wøjdemann M, Wettergren A, Hartmann B, Holst JJ. Glucagon-like peptide-2 inhibits centrally induced antral motility in pigs. Scand J Gastroenterol 1998;33:828-32.

34. Muskhelishvili L, Latendresse JR, Kodell RL, Henderson EB. Evaluation of cell proliferation in rat tissues with BrdU, PCNA, Ki-67(MIB-5) immunohistochemistry and in situ hybridization for histone mRNA. J Histochem Cytochem 2003;51:1681-8.

35. Watanabe M, Hitomi M, van der Wee K, et al. The pros and cons of apoptosis assays for use in the study of cells, tissues, and organs. Microsc Microanal 2002;8:375-91.

36. Potten CS. Epithelial cell growth and differentiation. II. Intestinal apoptosis. Am J Physiol 1997;273(2 Pt 1):G253-7.

37. Potten CS. Stem cells in gastrointestinal epithelium: numbers, characteristics and death. Philos Trans R Soc Lond, B, Biol Sci 1998;353: 821-30.

38. Varedi M, Chinery R, Greeley GH Jr, Herndon DN, Englander EW. Thermal injury effects on intestinal crypt cell proliferation and death are cell position dependent. Am J Physiol Gastrointest Liver Physiol 2001;280:G15763.

39. Shin ED, Estall JL, Izzo A, Drucker DJ, Brubaker PL. Mucosal adaptation to enteral nutrients is dependent on the physiologic actions of glucagon-like peptide-2 in mice. Gastroenterology 2005;128:1340-53.

40. Ivory CP, Wallace LE, McCafferty DM, Sigalet DL. Interleukin-10-independent anti-inflammatory actions of glucagon-like peptide 2. Am J Physiol Gastrointest Liver Physiol 2008;295:G1202-10. 